\title{
EHMTI-0291. Chronic headache is associated with mental vulnerability, depression, and neuroticism and poor mental health-related quality of life: a cross-sectional population study
}

\author{
S Ashina ${ }^{1 *}$, AC Lyngberg ${ }^{2}$, L Bendtsen ${ }^{3}, D$ Buse ${ }^{4}$, RB Lipton ${ }^{4}, R$ Jensen ${ }^{3}$ \\ From 4th European Headache and Migraine Trust International Congress: EHMTIC 2014 \\ Copenhagen, Denmark. 18-21 September 2014
}

\section{Introduction}

Psychiatric comorbidity in migraine and tension-type headache (TTH) is well established.

\section{Aim}

To study differences in mental health variables in relation to headache subtype and frequency.

\section{Methods}

A sample of 547 subjects completed a questionnaire based on ICHD-1 and provided data on mental vulnerability (12-item scale), depression (MDI), neuroticism (Eysenck Personality Questionnaire) and mental health-related quality of life (SF-12). Results were adjusted for age, gender and education in a multiple regression model. Chronic headache indicated headache on $\geq 15$ days per month.

\section{Results}

Mental vulnerability scores (mean $\pm \mathrm{SD}$ ) were highest for chronic headache (migraine $+/-$ TTH) headache $(6.1 \pm$ 1.9), followed by chronic TTH (5.7 \pm 2.1$)$, episodic migraine +/- TTH ( $4.2 \pm 1.9)$, episodic TTH $(4.2 \pm 1.6)$, no headache $(3.1 \pm 1.4),(\mathrm{p}<0.001)$. Neuroticism scores were highest for chronic TTH $(11.7 \pm 8.2)$, followed by chronic headache $(9.5 \pm 4.9)$, episodic TTH $(8.9 \pm 4.9)$, episodic migraine $+/$ - TTH $(8.8 \pm 4.5)$, no headache $(6.2 \pm$ $4.3),(p<0.001)$. Depression scores were highest for chronic headache $(13.8 \pm 10.4)$, followed by chronic TTH $(12.6 \pm$ $14.0)$, episodic migraine $+/$ - TTH $(7.4 \pm 6.3)$, episodic TTH (7.2 \pm 7.3$)$, and no headache $(4.4 \pm 4.9),(\mathrm{p}<0.001)$.
SF-12 scores were lowest in chronic TTH $(43.0 \pm 11.6)$, followed by chronic headache $(48.1 \pm 10.1)$, episodic TTH (51.2 \pm 8.8$)$, episodic migraine $+/-$ TTH $(50.4 \pm 8.0)$, no headache $(53.9 \pm 6.8),(\mathrm{p}<0.001)$.

\section{Conclusions}

Chronic migraine and TTH areis associated with low mental health-related quality of life, high mental vulnerability, depression and neuroticism scores.

\section{Authors' details}

${ }^{1}$ Neurology, Mount Sinai Beth Israel Icahn School of Medicine at Mount Sinai, New York, USA. ${ }^{2}$ Unit for Quality and Patient Safety, Capital Region of Denmark, Hvidovre, Denmark. ${ }^{3}$ Neurology, Danish Headache Center University of Copenhagen Glostrup Hospital, Glostrup, Denmark. ${ }^{4}$ Neurology, Montefiore Headache Center Albert Einstein College of Medicine, New York, USA.

Published: 18 September 2014

\section{doi:10.1186/1129-2377-15-S1-B2}

Cite this article as: Ashina et al: EHMTI-0291. Chronic headache is associated with mental vulnerability, depression, and neuroticism and poor mental health-related quality of life: a cross-sectional population study. The Journal of Headache and Pain 2014 15(Suppl 1):B2. 